\title{
Outcome of functional end-to-end esophagojejunostomy in totally laparoscopic total gastrectomy
}

\author{
Yuma Ebihara • Shunichi Okushiba • Yo Kawarada • \\ Shuji Kitashiro • Hiroyuki Katoh
}

Received: 6 September 2012 / Accepted: 14 January 2013 / Published online: 25 January 2013

(C) The Author(s) 2013. This article is published with open access at Springerlink.com

\begin{abstract}
Purpose Totally laparoscopic total gastrectomy (TLTG) is unpopular because reconstruction is difficult. In fact, esophagojejunostomy is the most difficult surgical technique in TLTG. We adopted functional end-to-end anastomosis for esophagojejunostomy to simplify the procedure. The present study assesses the feasibility and surgical outcomes of TLTG with functional end-to-end esophagojejunostomy.

Methods We assessed the intraoperative and postoperative outcomes of 65 consecutive patients who underwent TLTG with functional end-to-end esophagojejunostomy at Tonan Hospital between January 2006 and August 2011.

Results The mean surgical duration was $271.5 \pm 64.7 \mathrm{~min}$, and the mean blood loss was $85.2 \pm 143.2$ g. One patient $(1.5 \%)$ was converted to open surgery, and two patients $(3.1 \%)$ required reoperation due to ileus because of an internal hernia and jejunojejunostomy leakage. No reoperation was associated with functional end-to-end esophagojejunostomy. The mean hospital stay was $21.4 \pm 13.5$ days. Ten patients $(15.4 \%)$ developed postoperative complications, of which three (4.6\%) were anastomotic stenosis associated with functional end-to-end esophagojejunostomy. All of these were resolved by endoscopic dilation. Conclusion Functional end-to-end esophagojejunostomy in TLTG is safe and feasible.
\end{abstract}

Keywords Gastric cancer · Totally laparoscopic gastrectomy · Total gastrectomy · Functional end-to-end esophagojejunostomy

Y. Ebihara $(\bowtie) \cdot$ S. Okushiba $\cdot$ Y. Kawarada $\cdot$ S. Kitashiro $•$

H. Katoh

Department of Surgery, Tonan Hospital, N1W6, Chuuou-ku,

Sapporo, Hokkaido 060-0001, Japan

e-mail: yuma-ebi@wc4.so-net.ne.jp

\section{Introduction}

Since the first laparoscopy-assisted distal gastrectomy for early gastric cancer was performed in 1991 [1], the development of dedicated instruments and surgical techniques has led to laparoscopic distal gastrectomy (LDG) to treat gastric cancer. However, laparoscopic total gastrectomy (LTG) for gastric cancer has not become as popular as LDG, because reconstruction is more complex after LTG than after LDG, especially for esophagojejunostomy. Construction of an esophagojejunostomy is technically difficult, and serious complications can arise. Use of a circular stapler can be technically challenging during laparoscopic procedures for obese patients and for those with a relatively narrow esophagus. Some surgeons have used an extracorporeal approach through a mini-laparotomy, constructing an end-to-side anastomosis with an end-to-end anastomosis stapler [2]. One recent report has described LTG using a transorally inserted anvil for esophagojejunostomy [3]. However, an optimal procedure for esophagojejunostomy has yet to be established.

The entire anastomotic procedure can be clearly viewed during totally LTG (TLTG), and thus, tension and damage can be prevented. We have performed 65 TLTG procedures using functional end-to-end anastomosis for esophagojejunostomy since January 2006. This article describes the intraoperative manipulations and postoperative outcomes of TLTG with functional end-to-end esophagojejunostomy.

\section{Materials and methods}

\section{Patients}

We enrolled 65 consecutive patients who underwent TLTG with functional end-to-end esophagojejunostomy at Tonan 
Hospital between January 2006 and August 2011. The eligibility criterion was clinical stage IA or IB gastric cancer that was preoperatively diagnosed by endoscopy, computed tomography, and endoscopic ultrasound. All of the patients provided written informed consent to participate in the study. Specimens were evaluated basically according to the Japanese Classification of Gastric Carcinoma established by the Japanese Research Society for Gastric Cancer [4].

\section{Surgical technique}

Patients are placed in the supine reverse Trendelenburg position with the legs apart under general anesthesia. Five Exel trocars (Ethicon Endo-Surgery, Cincinnati, OH, USA) are used, and a $12-\mathrm{mm}$ paraumbilical port is subsequently extended to $3.0 \mathrm{~cm}$ when extracting specimens. After carbon dioxide pneumoperitoneum is achieved at a pressure of $10 \mathrm{mmHg}$, an electrolaparoscope (WA50013, Olympus Medical Systems, Tokyo, Japan) is introduced through this port, and the four other trocars (three $12 \mathrm{~mm}$ and one $5 \mathrm{~mm}$ ) are positioned as shown in Fig. 1. We dissect lymph nodes and coagulated vessels using laparoscopic coagulation shears (SONOSURG-X, Olympus Medical Systems or Harmonic Ace, Ethicon Endo-Surgery). The basic extent of lymph node dissection in the present series was D1+, but patients with clinical N1 underwent D2+ splenectomy, both of which were achieved using TLTG [4].

\section{Intracorporeal anastomosis with TLTG}

Figure 2 shows a functional end-to-end esophagojejunostomy in TLTG. The abdominal esophagus is exposed and transected using an Echelon 60 Endopath 60-mm linear stapler (Ethicon Endo-Surgery, Cincinnati, OH, USA) in the horizontal direction. Thereafter, $10-\mathrm{mm}$ transverse incisions are created at the edges of the tip of the antimesenteric border between the

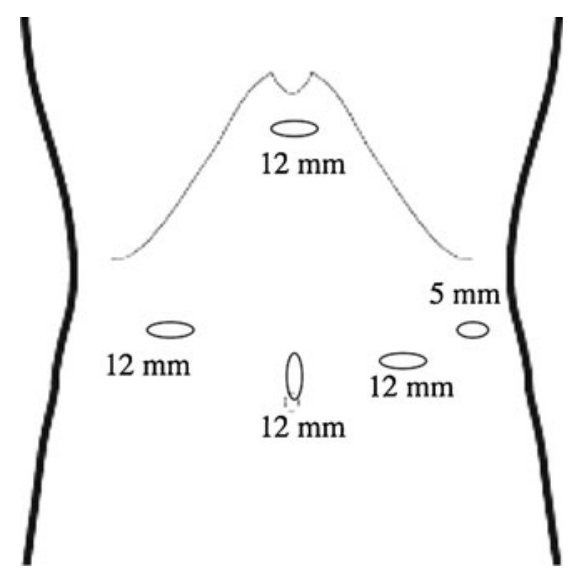

Fig. 1 Positions of surgical ports. Four 12-mm trocars are placed in paraumbilical, bilateral abdominal, and epigastric regions. One 5-mm trocar is placed in the left hypochondrial area
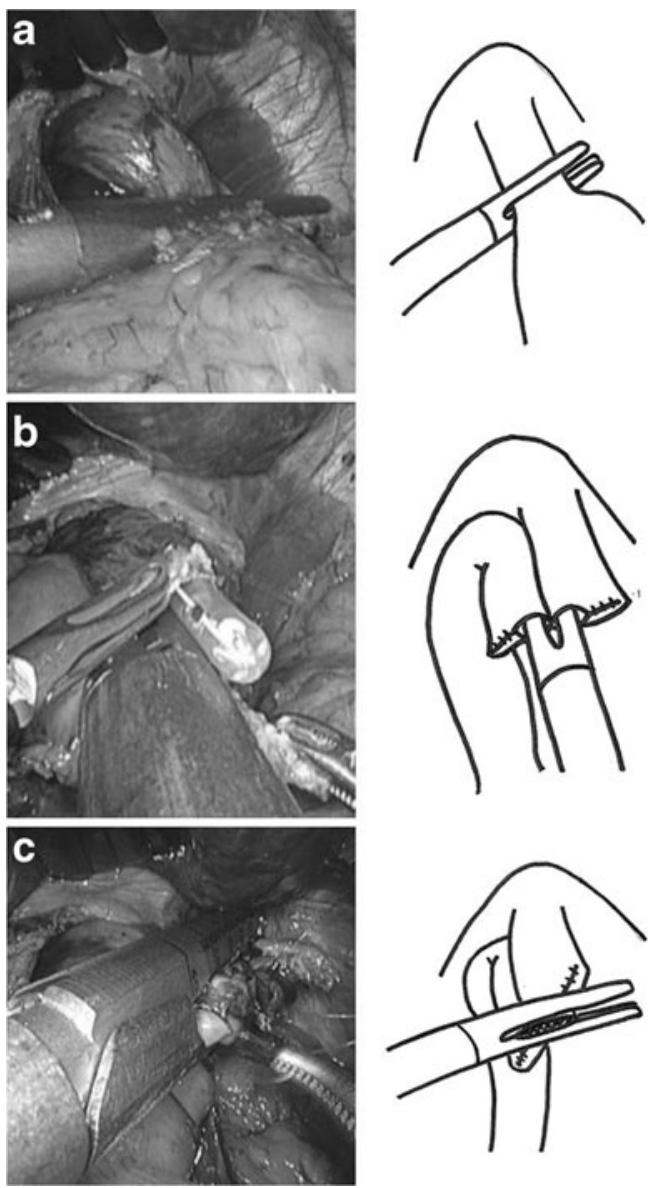

Fig. 2 Functional end-to-end esophagojejunostomy in totally laparoscopic total gastrectomy. a Abdominal esophagus is transected using 60-mm linear stapler. b Both jaws of 45-mm linear stapler are inserted into holes and fired. c Entry hole is closed using $60-\mathrm{mm}$ linear stapler

jejunum and the right lateral wall of the abdominal esophagus. Both jaws of a 45-mm linear stapler (ETS Flex45, Ethicon Endo-Surgery, Cincinnati, OH, USA) are inserted into holes and fired. The entry hole for the Echelon 60 Endopath linear stapler is closed with one application of the stapler perpendicular to the first suture line. Functional end-to-end esophagojejunostomy is then accomplished. Some hand sutures are added for reinforcement, depending upon the conditions of the anastomosis. A side-to-side jejunostomy proceeds at a point $40 \mathrm{~cm}$ distal to the esophagojejunostomy using the ETS Flex45 linear stapler. The entry hole for this stapler is closed with a running suture (3-0 Vicryl, Ethicon). When we find a narrow esophageal hiatus that diminishes the working space during laparoscopic functional end-to-end esophagojejunostomy, we extend the hiatus to incise the diaphragm in the ventral direction because incising the esophageal crus to extend the hiatus can generate pneumothorax. We use a $45-\mathrm{mm}$ linear stapler because we achieved functional end-to-end esophagojejunostomy using a $60-\mathrm{mm}$ linear stapler during the early period of TLTG in three patients with anastomotic stenosis. The $60-\mathrm{mm}$ linear stapler lacks grasping power, perhaps because the 
esophageal mucosa can slip off and become shortened before firing. Moreover, we occasionally found that the abdominal esophagus does not stretch during functional end-to-end esophagojejunostomy with a $60-\mathrm{mm}$ linear stapler.

\section{Clinicopathological findings}

Information about the patients including gender, age, body mass index (BMI), operative duration, blood loss, number of lymph node dissections, pathological findings, and perioperative complications was collected from the medical and anesthesia records.

All values are expressed as means \pm standard deviation.

\section{Results}

Table 1 shows the clinical features of the patients. The mean age was $65.9 \pm 10.2$ years, and $45(69.2 \%)$ male and 20 (30.8\%) female patients underwent TLTG with functional end-to-end esophagojejunostomy. Their mean BMI was $23.5 \pm 4.0 \mathrm{~kg} / \mathrm{m}^{2}$. The upper and middle regions of the stomach were affected by malignancies in $53(81.5 \%)$ and $12(18.5 \%)$ patients, respectively. The clinical stages were stages IA and IB in $41(63.1 \%)$ and $24(36.9 \%)$ patients, respectively. Table 2 shows details of the surgical and pathological data. The mean surgical duration was $271.5 \pm 64.7 \mathrm{~min}$, and the mean blood loss was $85.2 \pm 143.2 \mathrm{~g}$. One $(1.5 \%)$ patient was converted to open surgery due to low blood pressure of uncertain cause during the procedure. The type of lymph node dissection was $\mathrm{D} 1+$ in $62(95.4 \%)$ patients and D2+ splenectomy in $3(4.6 \%)$ patients, and 30.2 \pm 12.4 lymph nodes were dissected. The cancer had invaded the mucosa, submucosa, muscle, subserosa, and serosa in 18 (27.7\%), 28 (43.1\%), 9 (13.8\%),

Table 1 Clinical features of patients who underwent TLTG

Values in parentheses are percentages

$B M I$ body mass index, ASA American Society of Anesthesiologists Physical Status

${ }^{\mathrm{a} M e a n s}$ (standard deviation)
Table 2 Operative and pathological data

\begin{tabular}{ll}
\hline Variable & TLTG $(n=65)$ \\
\hline Operation time (min) $^{\mathrm{a}}$ & $271.5(64.7)$ \\
Blood loss $(\mathrm{g})^{\mathrm{a}}$ & $85.2(143.2)$ \\
Conversion to open surgery $^{\mathrm{L}}$ & $1(1.5)$ \\
Lymph node dissection & \\
D1+ & $62(95.4)$ \\
D2+ (splenectomy) & $3(4.6)$ \\
No. of dissected lymph node & \\
Depth of cancer invasion & $30.2(12.4)$ \\
Mucosa & \\
Submucosa & $18(27.7)$ \\
Muscle & $28(43.1)$ \\
Subserosa & $9(13.8)$ \\
Serosa & $5(7.7)$ \\
Histological type & $5(7.7)$ \\
Well differentiated & \\
Moderately differentiated & $19(29.2)$ \\
Poorly differentiated & $16(24.6)$ \\
Lymph node metastasis & $30(46.2)$ \\
Absent & \\
Present & $53(81.5)$ \\
\hline
\end{tabular}

Values in parentheses are percentages

${ }^{a}$ Means (standard deviation)

$5(7.7 \%)$, and $5(7.7 \%)$ patients, respectively. The histological type was well, moderately, and poorly differentiated in 19 (29.2\%), $16(24.6 \%)$, and $30(46.2 \%)$ patients, respectively. Twelve $(18.5 \%)$ patients had lymph node metastasis. Table 3

Table 3 Postoperative outcomes

\begin{tabular}{ll}
\hline Variable & TLTG $(n=65)$ \\
\hline Postoperative complication & $10(15.4)$ \\
EJ leakage & 0 \\
JJ leakage & $1(1.5)$ \\
DS leakage & $1(1.5)$ \\
EJ stenosis & $3(4.6)$ \\
Pancreatic juice fistula & $2(3.1)$ \\
Paralytic ileus & $1(1.5)$ \\
Wound infection & $2(3.1)$ \\
EJ anastomosis-related complications & $3(4.6)$ \\
Reoperation & $2(3.1)$ \\
Operation-related death & $1(1.5)$ \\
Time to first flatus (days) & $1.9(0.7)$ \\
Time to resume soft diet (days) & a \\
Postoperative hospital stay (days) & \\
\hline
\end{tabular}

Values in parentheses are percentages

$E J$ esophagojejunostomy, $J J$ jejunojejunostomy, $D S$ duodenal stump

${ }^{\mathrm{a}}$ Values are means (standard deviation) 
shows the postoperative outcomes. Ten $(15.4 \%)$ patients developed postoperative complications comprising jejunojejunostomy leakage in one $(1.5 \%)$, duodenal stump leakage in one $(1.5 \%)$, esophagojejunostomy stenosis in three (4.6\%), pancreatic juice fistula in two (3.1\%), paralytic ileus in one $(1.5 \%)$, and wound infection in two (3.1\%). No esophagojejunostomy leaked after TLDG, and related complications that developed in three $(4.6 \%)$ patients were resolved by endoscopic dilation. One patient who underwent reoperation for afferent loop syndrome died of septic shock on postoperative day 90 . The means of elapsed time until first flatus, resuming a soft diet, and discharge were $1.9 \pm 0.7,4.6 \pm 1.8$, and $21.4 \pm$ 13.5 days, respectively. Cancer did not recur in any of the patients during a median follow-up of 37 (11-68) months.

\section{Discussion}

Laparoscopic surgery confers upon patients the advantages of faster recovery, fewer complications, reduced hemorrhaging that reduces the likelihood of needing a blood transfusion, a smaller incision that reduces pain, the probability of intestinal obstruction, and the risk of wounding. Since its introduction in 1994, LDG for gastric cancer has become a commonplace due to the development of dedicated instruments and surgical techniques [1]. Many surgeons have described their experiences with totally laparoscopic gastrectomy and have found it safe and feasible [5-8]. Kim et al. concluded that TLDG helps to improve early surgical outcomes [9]. A policy of totally laparoscopic gastrectomy was adopted at our hospital from its inception because we considered that it would confer several advantages. Extracorporeal anastomosis via mini-laparotomy incisions during laparoscopy-assisted gastrectomy could cause forceful tension and damage structures around an anastomosis because of limited vision, especially in obese patients $[6,10]$. The entire anastomotic procedure can be clearly viewed during totally laparoscopic gastrectomy, which prevents such tension and damage. However, circular staplers are usually used for esophagojejunostomy in most LTG with mini-laparotomy incisions $[11,12]$. The intra-abdominal application of a circular stapler might be challenging, and intracorporeal purse-string sutures and anvil placement are required for obese patients as well as for those with a relatively narrow esophagus $[11,13]$. Okabe et al. noted that a circular stapler has not been specifically designed for endoscopic surgery and such staplers have usually been extracorporeally applied because of difficulties with applying them under a limited laparoscopic view [14]. If force is applied to a relatively large anvil, the mucosa can slip off and rupture the esophageal wall, which would result in serious complications [15]. In contrast, a linear stapler can be easily manipulated intra-abdominally. Steichen introduced a functional end-toend anastomosis that has been adopted worldwide [16].
Matsui et al. and Lee et al. concluded that functional end-toend esophagojejunostomy after a total gastrectomy is convenient, safe, reliable, and independent of the esophagus and depth of the esophageal hiatus [17, 18]. Functional end-to-end esophagojejunostomy after total gastrectomy in open surgery has become accepted, whereas laparoscopic esophagojejunostomy seems quite rare. We adopted this technique from the introduction of TLTG at our hospital. We have not experienced esophagojejunostomy leakage, and esophagojejunostomy stenosis that developed in three $(4.6 \%)$ patients was resolved by endoscopic dilation, the frequency of which varies from 3 to $10 \%$ when a circular stapler is applied $[19,20]$. One of our patients died in the hospital due to afferent loop syndrome caused by an internal hernia, which is a potentially serious complication after TLTG. Because of this experience, we close mesenteric defects using an intracorporeal suture. We do not apply this method to patients with tumors that have invaded the esophagus, and we perform intracorporeal circular stapling for esophagojejunostomy using a transorally inserted $\mathrm{OrVil}^{\mathrm{TM}}$ anvil (Covidien, Mansfield, MA, USA). If a tumor is close to the esophago-cardiac junction, we transect the abdominal esophagus before starting an esophagojejunostomy. We have not experienced anastomotic stenosis since using the 45-mm linear stapler, which has more grasping power. As a result, the suture line between the esophageal and the intestinal wall does not shift. The long-term outcomes of functional end-to-end esophagojejunostomy in TLTG remain unknown, and thus, further clinical trials and prospective controlled studies are required for comparisons with other types of anastomosis. We achieved functional end-to-end esophagojejunostomy in TLTG without anastomotic leakage and consider this technique safe and feasible.

Conflicts of interest All authors state that they have no commercial associations that might pose a conflict in connection with the submitted article.

Open Access This article is distributed under the terms of the Creative Commons Attribution License which permits any use, distribution, and reproduction in any medium, provided the original author(s) and the source are credited.

\section{References}

1. Kitano S, Iso Y, Moriyama M, Sugimachi K (1994) Laparoscopyassisted Billroth I gastrectomy. Surg Laparosc Endosc 4:146-148

2. Asao T, Hosouchi Y, Nakabayashi T, Haga N, Mochiki E, Kuwano H (2001) Laparoscopically assisted total or distal gastrectomy with lymph node dissection for early gastric cancer. Br J Surg 88:128-132

3. Jeong O, Park YK (2009) Intracorporeal circular stapling esophagojejunostomy using the transorally inserted anvil (OrVil ${ }^{\mathrm{TM}}$ ) after laparoscopic total gastrectomy. Surg Endosc 23:2624-2630

4. Japanese Gastric Cancer Association (1998) Japanese Classification of Gastric Carcinoma-2nd English Edition. Gastric Cancer 1:10-24 
5. Kanaya S, Gomi T, Momoi H, Tamaki N, Isobe H, Katayama T, Wada Y, Ohtoshi M (2002) Delta-shaped anastomosis in totally laparoscopic Billroth I gastrectomy. J Am Coll Surg 195:284-287

6. Kim JJ, Song KY, Chin HM, Kim W, Jeon HM, Park CH, Park SM (2008) Totally laparoscopic gastrectomy with various types of intracorporeal anastomosis using laparoscopic linear staplers: preliminary experience. Surg Endosc 22:436-442

7. Song KY, Park CH, Kang HC, Kim JJ, Park SM, Jun KH, Chin HM, Hur H (2008) Is totally laparoscopic gastrectomy less invasive than laparoscopy-assisted gastrectomy?: prospective, multicenter study. J Gastrointest Surg 12:1015-1021

8. Tanimura S, Higashino M, Fukunaga Y, Takemura M, Nishikawa T, Tanaka Y, Fujiwara Y, Osugi H (2008) Intracorporeal Billroth I reconstruction by triangulating stapling technique after laparoscopic distal gastrectomy for gastric cancer. Surg Laparosc Endosc Percutan Tech 18:54-58

9. Kim MG, Kawada H, Kim BS, Kim TH, Kim KC, Yook JH, Kim BS (2011) A totally laparoscopic distal gastrectomy with gastroduodenostomy (TLDG) for improvement of the early surgical outcomes in high BMI patients. Surg Endosc 25:1076-1082

10. Choi YY, Kim YJ (2011) Intracorporeal anastomosis using a Lapra-ty clip in laparoscopic distal gastrectomy: initial clinical experiences. J Laparoendosc Adv Surg Tech A 21:51-55

11. Dulucq J, Wintringer P, Perissat J, Mahajna A (2005) Completely laparoscopic total and partial gastrectomy for benign and malignant diseases: a single institute's prospective analysis. J Am Coll Surg 200:191-197

12. Usui S, Yoshida T, Ito K, Hiranuma S, Kudo S, Iwai T (2005) Laparoscopy-assisted total gastrectomy for early gastric cancer: comparison with conventional open total gastrectomy. Surg Laparose Endosc Percutan Tech 15:309-314

13. Ziqiang W, ZhiMin C, Jun C, Xiao L, Huaxing L, PeiWu Y (2008) A modified method of laparoscopic side-to-side esophagojejunal anastomosis: report of 14 cases. Surg Endosc 22:2091-2094

14. Okabe H, Obama K, Tanaka E, Nomura A, Kawamura J, Nagayama S, Itami A, Watanabe G, Kanaya S, Sakai Y (2009) Intracorporeal esophagojejunal anastomosis after gastrectomy for patients with gastric cancer. Surg Endosc 23:2167-2171

15. Okushiba S, Kawarada Y, Shichinohe T, Manase H, Kitashiro S, Katoh H (2005) Esophageal delta-shaped anastomosis: a new method of stapled anastomosis for the cervical esophagus and digestive tract. Surg Today 35:341-344

16. Steichen FM (1968) The use of staplers in anatomical side-to-side and functional end-to-end enteroanastomoses. Surgery 64:948-953

17. Matsui H, Uyama I, Sugioka A, Fujita J, Komori Y, Ochiai M, Hasumi A (2002) Linear stapling forms improved anastomoses during esophagojejunostomy after a total gastrectomy. Am J Surg 184:58-60

18. Lee IS, Kim TH, Kim KC, Yook JH, Kim BS (2012) Modified techniques and early outcomes of totally laparoscopic total gastrectomy with side-to-side esophagojejunostomy. J Laparoendosc Adv Surg Tech A 22:876-880

19. Dorsey JS, Esses S, Goldberg M, Stone R (1980) Esophagogastrectomy using the auto suture EEA surgical stapling instrument. Ann Thorac Surg 30:308-312

20. Wong J, Cheung H, Lui R, Fan YW, Smith A, Siu KF (1987) Esophagogastric anastomosis performed with a stapler: the occurrence of leakage and stricture. Surgery 101:408-415 